\title{
Stem cells therapy for regenerative medicine: Principles of present and future practice
}

\author{
José A. Andrades ${ }^{1,2}$, José Becerra ${ }^{1,2,3}$, Ramón Muñoz-Chápuli ${ }^{4}$, Salvador Martínez ${ }^{5}$, Ángel Raya ${ }^{2,6,7}$, \\ Javier García-Sancho ${ }^{8}$, José M. Moraleda ${ }^{9}$ \\ ${ }^{1}$ Laboratory of Bioengineering and Tissue Regeneration (LABRET), Department of Cell Biology, Genetics and Physiology, Faculty \\ of Sciences, IBIMA, University of Málaga, Málaga, Spain \\ ${ }^{2}$ Networking Biomedical Research Center in Bioengineering, Biomaterials and Nanomedicine (CIBER-BBN), Málaga, Spain \\ ${ }^{3}$ Andalusian Centre for Biotechnology and Nanomedicine (BIONAND), Parque Tecnológico de Andalucía, Campanillas, Málaga, \\ Spain \\ ${ }^{4}$ Department of Animal Biology, Faculty of Sciences, University of Málaga, Málaga, Spain \\ ${ }^{5}$ Institute of Neurosciences, University of Miguel Hernández, Alicante, Spain \\ ${ }^{6}$ Control of Stem Cell Potency Group, Institut for Bioengineering of Catalonia (IBEC), Barcelona, Spain \\ ${ }^{7}$ Institució Catalana de Recerca i Estudis Avançats (ICREA), P. Lluís Companys, Barcelona, Spain \\ ${ }^{8}$ Institute of Molecular Biology and Genetics (IBGM), University of Valladolid, Valladolid, Spain \\ ${ }^{9}$ Unit of Transplant and Cell Therapy, University of Murcia, IMIB, University Clinic Hospital Virgen de la Arrixaca, El Palmar, \\ Murcia, Spain \\ Email: andrades@uma.es
}

Received 31 October 2013; revised 3 December 2013; accepted 13 December 2013

Copyright (C 2014 José A. Andrades et al. This is an open access article distributed under the Creative Commons Attribution License, which permits unrestricted use, distribution, and reproduction in any medium, provided the original work is properly cited. In accordance of the Creative Commons Attribution License all Copyrights (C) 2014 are reserved for SCIRP and the owner of the intellectual property José A. Andrades et al. All Copyright (C) 2014 are guarded by law and by SCIRP as a guardian.

\section{ABSTRACT}

The characterization and isolation of various stem cell populations, from embryonic to tissue-derived stem cells and induced pluripotent stem cells (iPSCs), have led to a rapid growth in the field of stem cell research and its potentially clinical application in the field of regenerative medicine and tissue repair. Stem cell therapy has recently progressed from the preclinical to the early clinical trial arena for a variety of diseases states, although further knowledge on action mechanisms, long-term safety issues, and standardization and characterization of the therapeutic cell products remains to be thoroughly elucidated. In this paper we summarize the current state of the art of basic and clinical research that was highlighted at the 2012 meeting of the Spanish Cell Therapy Network. This includes the current research involving genomic and transcriptomic characterization of selected stem cell populations, studies of the role of resident and transplanted stem cells during tissue regeneration and their mechanism of action, improved new strategies of tissue engineering, transplantation of mesenchymal stem cells (MSCs) in different animal models of disease, disease correction by iPSCs, and preliminary results of cell therapy in human clinical trials.

\section{KEYWORDS}

Spanish Cell Therapy Network; Stem Cell; Cell

Therapy; Tissue Engineering; Regenerative Medicine

\section{INTRODUCTION}

Stem cells are broadly defined as cells capable of going through numerous cycles of cell division, maintaining an undifferentiated state and having the capacity to differentiate into specialized cell types. The use of stem cells as a therapeutic tool for a number of ischemic, inflammatory, autoimmune, metabolic and degenerative diseases represents one of the more promising areas of biomedical research; however, after more than 15 years of an exponential increase in basic knowledge, the promise to translate all those findings to clinical practice has not been achieved [1]. There is only one cell therapy product that has obtained market authorization in the EU, and despite several hundreds of phase I-II clinical trials there is almost a complete lack of robust efficacy data from phase III trials. Currently there are a number of bottlenecks that prevent this translation, from the difficulties to establish business models that make these therapies attractive to industry, to the need of standardization of cell products, increase the understanding of mechanisms of action, or development of optimized new cell and tissue 
engineering products with improved performance [2].

The Spanish Cell Therapy Network (TerCel) has developed an intense activity during the last 10 years in order to tackle some of these questions and to build proofs of concept showing that cell therapy may have a positive impact on public health. TerCel is a research network organized by the Spanish National Institute of Health Carlos III that is composed of 33 clinical and basic research groups with more than 150 investigators. The aim of the net is to improve the knowledge about stem cells and its role in tissue repair and translate this research to the clinical practice by producing new cell-based and tissue-engineering treatments. The scientific scope of TerCel is distributed in three major research programs: cardiovascular diseases, neurodegenerative diseases, and a broad group of inflammatory, immune and metabolic diseases such as osteoarthritis, Crohn's disease and diabetes. The network has been quite successful with more than 20 registered clinical trials so far (www.redtercel.com).

Dr. Muñoz-Chápuli addresses the advances of the cardiovascular program; the investigators presented new insights in the molecular pathways during embryonic development and in cardiac regeneration. They also revealed new data on the generation of an in vitro model of cardiomyopathy, as well as the factors that may affect the regenerative potential of bone marrow stem cells, and new strategies for cardiac tissue engineering.

Dr. Martínez summarizes the advances in the Neurodegenerative Program. In this working party a wide range of advances were presented including the molecular regulation of the neurogenic niche, the characterization of new transcription factors for neural differentiation, routes of cell migration during development, mechanisms of neurotrophism-neuroprotection, and results of phase I-II clinical trials of cell therapy in brain ischemia, amyotrophic lateral sclerosis and Parkinson's disease.

Dr. Andrades deals with the advances presented in the osteo-articular workshop. Researchers presented data on the transcriptome of MSC from different sources, as well as the exo-fucosilation of bone marrow MSC to enhance engraftment in bone and inflammation sites. Results of several preliminary trials of MSCs for bone, cartilage and tendon regeneration were also discussed.

Dr. García-Sancho reviewed the implications of cell production for human use under GMP standards and the use of MSCs in several pilot trials in osteoarticular diseases such as knee osteoarthritis, degenerative disc disease or maxillary cysts.

Finally, Dr. Raya summarized the new strategies based on embryonic stem cells and iPSCs and discussed the possibilities and limitations of this fascinating approach for studying disease pathogenesis, discovering new molecular targets and assaying new ways of healing diseases.

The goal of regenerative medicine is to promote organ repair and regeneration, thus obviate the need for replacement. Stem cell therapy may participate in this process via paracrine mechanisms enhancing the local reparative machinery. The successful translation to the clinical practice requires an understanding of disease pathogenesis and stem cell biology, and a multidisciplinary approach in collaboration with other disciplines such as medical device technology, biomaterials science, gene therapy, and transplantation immunology. It is also essential for this progress to include the patients in carefully designed and ethically approved clinical trials, and a close linkage among the academy, the industry and the regulatory authorities.

The annual meeting of the net was held at the University of Málaga in October 2012 and this paper highlights the main scientific contributions in the three research programs of TerCel.

\section{CARDIOVASCULAR}

The first session of the TerCel 2012 meeting was devoted to show recent results from the nodes involved in cardiovascular research. The group led by Antonio Bernad in the CNIC (Madrid) presented results on Bmi1, a critical component of a Polycomb group (PcG) multiprotein PRC1-like complex, a transcriptional repressor of many genes throughout development. Bmi1 is a main regulator of cellular self renovation in the neural and hematopoietic systems. A cell lineage tracking using a Bmi1IresCreER model allowed this group to show that the adult murine heart contains a population of cardiac stem cells associated to Bmi1 expression. These cells are Sca-1+, but lack of c-Kit and CD45 expression. Clusters of Bmi1-CSCs appear in compact structures, scattered throughout the heart. The group has traced the progeny of these cells throughout the main cardiac cell lineages (cardiomyocytes, endothelium and smooth muscle) and it has shown evidence of the contribution of Bmi1-CSCs in cardiac homeostasis and also in response to injury.

The group of Felipe Prósper (CIMA, Pamplona) showed results on the effect of the adipose tissue mesenchymal stem (ATMS) cells on a model of myocardial infarct in rat, when they are implanted associated to biocompatible and biodegradable collagen matrices. This association proved to be better as compared with the implant of ATMS cells alone, improving ATMS cell surviving and cardiac functional parameters. This beneficial effect of the collagen matrices was also confirmed in a preclinical study on pigs. On the other hand, the group presented results on the therapeutic effects of FGF-1 and neuregulin-1, released by biocompatible and biodegradable microparticles of the copolymer PLGA (poly lactic coglycolic acid) in a model of myocardial infarction. The slow release of these factors stimulated the endogenous reparative mechanisms of the heart, reducing the infarct 
size and the fibrosis, and increasing revascularization and cardiomyocyte proliferation.

The group led by José L. de la Pompa (CNIC, Madrid) described an in vitro model for left ventricular noncompaction (LVNC), a cardiomyopathy whose molecular mechanisms are still unclear. Mutation in the Mind Bomb-1 gene (MIB1) caused LVNC in mice, as demonstrated by the group. Thus the Notch pathway revealed to be involved in LVNC, since MIB1 is a regulator of this signaling system. The group is currently generating induced pluripotent stem cells (iPSCs) from LVNC-affected patients carrying mutations in the MIB1 gene (Val943Phe and Arg530Stop). By differentiation of these cells into the main cardiac lineages (cardiomyocytes, endothelium, valve mesenchymal cells) it will be possible to generate an in vitro model to study the cellular and molecular mechanisms leading to LVNC and to discover and validate new therapeutic targets. This group also showed results about the role played by the Notch signaling pathway in zebrafish fin regeneration, particularly in the proliferation of the blastema cells and the maintaining of a pool of undifferentiated cells in the distal part of the regenerating fin.

The group of Lina Badimón (CIC Hospital Sant Pau, Barcelona) showed how cardiovascular disease risk factors affected bone marrow cells, decreasing their regenerative potential. Thus, the risk of thrombosis in Zucker diabetic fatty (ZDF) rats, which is higher than in normoglycemic controls, decreases significantly when ZDF rats receive transplants of bone marrow cells from normoglycemic rats, while bone marrow cells from ZDF transplanted to control rats increased their risk of thrombosis. ZDF rats also showed increased megakaryopoiesis and alterations in endoplasmic reticulum stress proteins (GRP78, PDI). Bone marrow cells from ZDF rats presented a significant downregulation of the canonical Wnt/ b-catenin signaling pathway and the expression of stem cell markers, associated to an increase of FOXO transcription factors. In conclusion, cardiovascular disease risk factors induce alterations in bone marrow cells reducing their regenerative potential and increasing the risk of thrombosis.

The group of Nadia Mercader (CNIC, Madrid) presented results on the fate of the epicardial-derived cells in cardiac regeneration in a zebrafish model. Using transplants of cardiac tissue enriched in epicardial cells they demonstrated that epicardial-derived cells contribute to the transient fibrotic response to injury of the zebrafish heart, but they are not involved in cardiomyocyte differentiation.

Finally, the group of José M. Pérez-Pomares and Ramón Muñoz-Chápuli (University of Málaga) presented results of a study about the lineage of the cells expressing the transcription factor Wt1 (suppressor of the Wilms tumor) and the contribution of this cell lineage to multiple tissues throughout development. In the heart, $\mathrm{Wt1}$ is expressed in the epicardium, thus the tracing of the $\mathrm{Wt} 1$ lineage through a mWt1/IRES/GFP-Cre model allows for identification of the epicardial-derived cells and their contribution to the coronary vasculature and cardiac interstitium. This model shows a large expansion of the Wt1-expressing cell lineage after myocardial infarction. The group has also characterized an immortalized mouse embryonic epicardial-derived cell line (EPIC) that can be useful for in vitro studies of the cardiac interstitium.

\section{NEURODEGENERATION}

Cell-mediated functional restoration in the human brain constitutes a challenging goal of current regenerative medicine. Cell therapy has emerged as a potential solution for the repair of the highly sophisticated functions of the central nervous system (CNS) that are lost in traumatic injuries or neurodegeneration. The interest of cell therapy approaches in neurology is even more undeniable in light of the progressive increase in the incidence of ageing-associated neurodegenerative disorders [3] have estimated that, in 2010, one third of European citizens were affected by one neural-related disease, with an economic cost of nearly 800 billion euros; more than cardiac and oncological pathologies together. Although the design of therapeutic strategies in neuroregenerative medicine is similar to those applied to other systems, experience accumulated in the recent years, both in animal models and clinical trials, have indicated that:

1) Transplantation of cells in the brain parenchyma seems to lead little functional integration of new neurons into the pre-existing circuits. Although, neurons differentiated from transplanted cells can degenerate faster than endogenous neurons or exhibit neurodegenerative signs characteristic of the diseased host brain [4,5], transplanted cells seem to exert, however, significant local influences and on the sustainability of endogenous neurons. Therefore, it is becoming well accepted that cell transplantation can contribute to the maintenance of neural functions by replacing lost cells and, more importantly, by activating local neurotrophic processes or by modulation of the inflammatory response [6].

2) Recent experiments suggest that endogenous neural stem cells (NSCs) are altered in neurodegenerative processes and that they can be activated by brain insults [7-9]. Therefore, understanding the mechanisms that regulate endogenous stem cell responses in the healthy and pathological brain may be required to design more efficient restorative responses.

In Málaga’s TerCel meeting, several groups exploring neurorestorative and neurotrophic potentialities of cell therapy in the Central Nervous System presented their results and progresses: 
- José M. García-Verdugo’s group (University of Valencia) described the anatomy and structure of the rostral migratory stream (RMS) in the developing human brain. This migratory stream of cells expressing Psa-NCAM, DCX and Tuj-1 can be followed from the lateral ventricle towards the olfactory bulbs in fetal and perinatal human brains (15 - 18 postnatal months), corresponding to the RMS described in the adult rodent brain. Moreover, although other migrations from the lateral subventricular zone have been also observed, their final targets of are not jet identified.

- Isabel Fariñas' group (University of Valencia) in collaboration with the Ferguson-Smith's group (University of Cambridge) presented original data on the differential control of insulin growth factor 2 (Igf2), ans its imprinted state, in adult neurogenic niches. They demonstrated that Igf2 signal can regulate neurogenesys of adult brain subventricular and subgranular neurogenic zones in the olfactory bulb and hippocampus, respectively. The imprinted character of Igf2 may represent mechanisms of functional adaptation by epigenetic regulation of adult neurogenesis.

- Josep M. Canals’ group (University of Barcelona) talk described that neuronal differentiation in the striatum is a process that requires the expression of the transcription factors Ikaros and Helios. Both genes are expressed in the lateral ganglionic eminence (LGE; striatal primordium) during embryonic development (E14,5-P15) and co-localized with neuronal markers. To better understand the mechanisms of neurogenesis in striatal primordium they performed a molecular characterization or striatal neurogenic region at different developmental stages. These results are showing new genes coding for structural molecules and signaling pathways involved in the development of striatal neurons.

- Juan J. Toledo-Aral's group (IBiS, University of Seville) revised the most recent data on experimental approaches using stem cells to reduce neurological symptoms of Parkinson Disease (PD). This group studied the potential therapeutic efficiency and functional mechanisms of carotid body cell aggregates (CC) intrastriatal grafts in animal models of PD. They have shown that the grafted CC cell aggregate generates a trophic effect more than nigrostriatal dopamine release. In fact, adult rodents CC express a neurotrophic factor: glial cell derived neurotrophic factor (GDNF). The expression of GDNF in glomus cells remains after intrastriatal transplantation in PD animal models. Therefore, CC may represent a durable and stable biological pump of GDNF, which can be used in cell therapy against PD. Actually, these studies allowed to propose and develop two clinical trials conducted to verify the feasibility of the CC cells autologous transplant in humans with PD. In these pilot some transplant patients showed both clinical and neurochemical improvement, suggesting that autologous CC intrastriatal graft could be a new therapeutic strategy, safe and feasible for the treatment of PD.

- Salvador Martinez's group (University of Alicante) has analyze the effect that bone marrow and adipose mesenchymal stem cells (MSCs) grafts exert in a chronic demyelination and Friedrich ataxia mice models. As a result, oligodendrocyte progenitors were detected surrounding the graft in experimental demyelinating brain models, as well as increasing of dorsal root ganglia cells survival in vitro Friedrich ataxia cellular and animal models. This potential therapeutic effect was due to a selective production of trophic factors by the MSCs.

- Xavier Navarro's group (University of Barcelona) compared the therapeutic benefits and protective mechanisms of local transplantation of bone marrow MSC versus olfactory envolvent glial cells (OECs) in adult rat spinal cord injury. Through a comparative study of gene array they found that neural protection mediated by MSC or the CSG is due to different tissular processes. Among them, the tissue repair depends of angiogenesis, inflammation and improvement of stress responding mechanisms, as well as loss of cellular homeostasis. These reactive pathways vary depending on grafting time after injury, indicating a significant differential role of postlesional period. Moreover, signaling pathways involved in graft rejection are also differentially expressed regarding the grafting time after spinal cord injury.

- José M. Moraleda’s group (Arrixaca University Clinic Hospital; University of Murcia) described the results of the Phase I/II clinical trial to investigate the feasibility, safety, and effectiveness of autologous bone marrow mononuclear cells (BMSCs) intraspinal grafts in patients with amyotrophic lateral sclerosis (ALS) (EudraCT No. 2006-00309612 clinicaltrials.gov NCT00855400). BMSCs transplantation was performed at the level of T3 and T4 with a novel neurosurgical technique [10]. The grafted 11 patients were evaluated for safety variables and neurological functional scales of the ALS. The results have been recently published, confirming the intraspinal implantation procedure is feasible and safe [11]. To extend the safety and to explore efficacy a randomized, controlled placebo, single-blind, stratified clinical trial is now under progress (clinicaltrials.gov, NCT01254539).

Other TerCel groups performing research on cell therapy for nervous system pathologies have also presented their results:

- Juan A. Barcia’s group (San Carlos Clinic Hospital) as TerCell associate group, described results after adipose mesenchymal stem cells (ADSCs) grafts in models of brain ischemia in mouse. Their results suggest that ADSCs modulates inflammation, promotes endogenous neurogenesis and increases angiogenesis, without generating adverse effects. These data confirm the safety of 
ADSCs grafts and could represent a new therapy in brain ischemic processes.

- Finally, Thomas Schimmang, from Javier GarcíaSancho's group (University of Valladolid) studied the potential of human mesenchymal stem cells (hMSCs) to differentiate towards hair cells and auditory sensorial neurons. The results demonstrate the potential of hMSCs to give rise to inner ear sensory cells and highlight the importance of identifying treatment regimes that yield neural cell types capable of responding to differentiation cues.

\section{OSTEO-ARTICULAR}

With the same uncertainties than others, skeletal disorders are one of the more relevant chapters of the MSCs application. The abundance of extracellular matrix in those tissues and their special properties in each of them focus the development of skeletal tissue engineering strategies. MSCs, biomaterials and inductive agents are the central motives of the confluence of major scientific fields. Science and engineering come together in this exciting field of regenerative medicine.

Several skeletal clinical situations are taken advantage from tissue engineering. Between then, ligaments and tendons repairs, articular cartilage regeneration, nonunion bone fractures and spinal fusion are being addressed in the TerCel meeting. José A. Andrades' group presented results on sophisticate prosthesis of hollow poli-L-lactic (PLA) stranded which reproduce the biomechanical behavior of ligaments and tendons. The hole is filled with a combination of PLA microparticles, hyaluronic acid (HA) and bone marrow MSCs. The whole construct is coated with HA to avoid adherences with the surrounded tissues [12].

Osteoarthritic pathologies are being widely studied by several groups of TerCel. In this area, Dr. Andrades presented an international collaboration about the use of TGF-beta1 and BMP-7 to induce synthesis of a superficial zone protein of articular cartilage in cultures of muscle-derived mesenchymal cells (MDMSCs) [13]. Accordingly, these cells would be used to regenerate the superficial zone of damaged cartilage in tissue engineering.

Pseudoarthrosis following bone fracture repair is one of the most prevalent pathologies of long bone. Froilán Granero-Moltó's project use MSCs from periostium (PMSCs) to promote bone formation in tissue engineering compositions. Such as cells respond properly to the hypoxia and the hematoma environment in the process of natural fracture repair. Since those cells have in vitro high proliferation capability, express MSCs superficial markers and differentiate into the osteoblast lineage, the authors are studying their use in a pseudoarthrosis atrophic fracture nude rat model [14].

Tissue engineering procedures for spinal fusion is a technology worldwide because the use of auto and allograft in classical surgery has more than 35\% failure, beside morbidity and infections transmission via allograft. As all over the world [15], there are several teams in TerCel studying such as pathology using different tissue engineering constructs, almost always using bone marrow or fat derived MSCs on different scaffolds. In this meeting, the Salamanca's group presented preliminary results of a clinical trial phase I using tricalcium phosphate and MSCs, with posterolateral mechanical fixation.

In conclusion, tissue engineering strategies for skeletal disorders are very promise but more research are needed to standardized MSCs culture and optimize the best biomaterial for each application, in order to assure binding affinity to osteoinductive molecules and enable vascular ingrowth for functional integration.

\section{CELL PRODUCTION AND REGULATORY ASPECTS}

The production of cells for human use may become the bottleneck that limits both clinical research and therapeutic use. European legislation imposes the condition of medicine for the cells, and this forces production and handling under very stringent GMP conditions. MSC treatments are probably those that are closer to clinical use, especially in the osteoarticular area, where the distance from MSC to its final fate, chondrocyte and osteoblast, is shorter. It has been reported that: 1) MSCs differentiate to chondrocytes; 2) MSCs, when co-cultured with chondrocytes, stimulate proliferation of these cells as well as synthesis of extracellular matrix; 3) MSCs have an immunomodulatory effect and secrete anti-inflammatory cytokines; and 4) in animal models, MSCs favour cartilage healing. We shall review briefly preclinical evidence that supports the usefulness of MSC for osteoarticular repair and comment on practical examples that are under development by the research groups in our TerCel Network node. The diseases addressed to are those with maximal morbidity, osteoarthritis (OA) and degenerative disk disease (DDD).

DDD may cause severe low-back pain, a large public health problem with significant economic and life quality impact. Chronic cases often require surgery, which may lead to biomechanical problems and accelerated degeneration of the adjacent segments. Cell-based therapies may circumvent these problems and have exhibited encouraging results both in vitro and in animal studies. We have already published the results of a clinical trial with autologous MSCs in 10 patients, with confirmation of feasibility and security, remarkable improvement of pain and disability and a small but significant elevation of disc water content, revealed by MRI after 12 months of treatment [16].

$\mathrm{OA}$ is the most prevalent cause of joint pain, func- 
tional loss, and disability. It often becomes chronic, and conventional treatments have demonstrated only modest clinical benefits, whereas cell-based therapies have shown encouraging results, both in animal studies and a few human case reports. We designed a phase I-II pilot trial with autologous MSCs for knee OA that is still active (NCT01183728 in the clinicaltrials.gov database), and results after one year-treatment, which are now being analyzed, seem encouraging, following the same trends as the DDD assay.

The cellular product consisted of MSCs prepared from autologous bone marrow by a proprietary protocol approved by the Spanish Medicine Agency and named MSV (see details in [16]). Cells were purified, expanded for 3 weeks and checked for correct antigenic phenotype. For transport, cells were suspended at a density of $5 \times$ $10^{6} / \mathrm{ml}$ in Ringer-Lactate containing $5 \mathrm{mM}$ glucose and $0.5 \%$ human serum albumin and applied within 12 hours of harvesting. The number of cells used was $20 \times 10^{6}$ for DDD and $40 \times 10^{6}$ for OA.

We are also using MSV to promote bone generation in order to refill the loss of substance in maxillary cysts. In this case autologous MSV cells are grown over a cross-linked autologous serum scaffold (Patent Number, WO2008/119855) [17]. This tissue engineering product has been tested with excellent results to refill critical loses of substance in rat and sheep maxillary bone. A phase I-II clinical trial is now in course (NCT01389661).

We are presently investigating improvements in the processes of production, storage, targeting and application of MSCs for treatment of bone and joint diseases. Emphasis is also placed on allogeneic MSCs because 1) there is recent evidence suggesting the absence of adverse effects and, 2) its use would simplify and cheapen production, and this would increase fraction of the population susceptible to cell therapy. Use of allogeneic cells would widen the horizon for MSCs use to much larger layers of population, as it would simplify both procedure and logistics, and would improve renders. Drawbacks include the possibility of immune rejection as it is well known for transplants. However, MSCs seem to evade antigenic recognition and to inhibit immune system, this leading to immunologic tolerance [18]. Allogeneic MSCs have been used repeatedly without immune problems not only in animals [19] but also in humans, where several clinical trials using MSCs have been performed with no reports of serious adverse events [20]. We are presently performing a randomized and controlled phase I-II clinical trial studying the effects of allogeneic MSV on knee osteoarthritis (NCT01586312).

\section{INDUCED PLURIPOTENT STEM CELLS: THE NEW TOOL IN THE BOX}

The possibility to reprogram human somatic cells to pluripotency has now become commonplace among biomedical researchers and even for the general public. The notion of induced reprogramming to pluripotency has so swiftly permeated the vocabulary of scientists across a wide array of disciplines that it would appear that induced pluripotent stem (iPS) cells have been with us for decades. First generated from mouse cells in 2006 by Kazutoshi Takahashi and Shinya Yamanaka [21], iPS cells were recognized as the main scientific breakthrough of 2008 [22], and resulted in Yamanaka's receiving the Nobel Prize in Physiology or Medicine 2012, just 6 short years after their discovery. During this time, iPS cell technology has had a profound impact in our understanding of the mechanisms that control cell fate identity, as well as creating unprecedented opportunities for cell replacement therapies and for human disease modeling.

The disruptive notion generalized by iPS cell technology, i.e.: that transient ectopic expression of a few factors may indeed completely reprogram cell fate, has also contributed to revamping the entire field of cell reprogramming, which is currently not limited to the generation of pluripotent stem cells, but also extends to the direct cell fate conversion between different types of somatic cells [reviewed in 23,24]. In the case of iPS cells, reprogramming to pluripotency was originally achieved by transient ectopic expression of factors OCT4, SOX2, KLF4, and c-MYC (OSKM, also known as the "Yamanaka cocktail"), although slightly different combinations of factors have also been used, and c-MYC has since been shown to be dispensable [reviewed in 25]. The delivery methods to achieve ectopic expression of the reprogramming factors have evolved very rapidly as well toward clinically safer alternatives; from integrating retroviruses or lentiviruses to non-integrative viruses (adenovirus, Sendai virus...), non-viral DNA vectors, and mRNA or even direct protein transduction. In summary, the generation of human iPS cells has been perfected in a way that makes it currently possible to reprogram almost any type of differentiated somatic cell with no manipulation of their genome. Irrespective of the generation method used, fully-reprogrammed iPS cells are essentially indistinguishable from human embryonic stem (hES) cells by most (if not all) criteria, including cell colony morphology and growth dynamics, expression of pluripotency-associated transcription factors and surface markers, overall gene expression and epigenetic profiles, capacity for indefinite expansion in vitro while maintaining karyotype stability, and pluripotent differentiation ability into derivatives of the 3 main embryo germ layers.

iPS cell-based technologies have now been adopted by countless laboratories around the world for investigating the basic mechanisms of cell fate reprogramming, for developing in vitro cellular models of human diseases, and/or for designing strategies for cell replacement ther- 
apies. This technology has also had a significant and early presence among TerCel members [26]. In fact, the first lines of human iPS cells generated in Europe came from laboratories affiliated to TerCel [27]. The unique blend of basic, applied and clinical researchers that make up this network represents an excellent opportunity for tackling specific biomedical questions where iPS cell technology may be particularly applicable. A key advantage of induced cell reprogramming is the possibility of generating iPS cells from patients that carry the precise genetic variants, both known and unknown, which may contribute to the disease. Specifically, iPS cells can be generated from patients showing sporadic or familial forms of the disease. In the past few years, disease-relevant cell types have been generated from iPS cells representing a variety of monogenic early-onset diseases, and shown to display specific features of the disease in vitro [28-34]. Moreover, iPS cell-based models of neurodevelopmental and psychiatric disorders such as Rett syndrome and schizophrenia have also been described [35]. However, it was unclear until very recently whether iPS cell technology could be used to model diseases of non-monogenic, complex etiology and/or late onset. To address this issue, a multidisciplinary team composed by 8 research groups, half of them belonging to TerCel, took on the challenge of modeling Parkinson's disease, including both genetic and idiopathic forms of the disease [36]. The success of this project was highly illustrative in two respects: 1) it revealed that the power of iPS cellbased disease modeling could also be harnessed for investigating the mechanisms underlying age-related diseases of complex genetic influence; and 2) it provided a good example of how goal-oriented projects that leverage on the raw potential of networks such as TerCel may bring about fruitful and timely collaborative efforts.

Arguably the most ambitious biomedical application of iPS cells would be the generation of disease-free, specific cell types for autologous cell replacement therapies. Proof-of-principle for this type of approach was provided in the context of Fanconi anemia patients by members of TerCel [37], in a study that also highlighted a number of important limitations for its clinical translation. Some of these limitations have been proven to be ultimately of technical nature and, indeed, have been successfully addressed in the last few years, including the presence of randomly integrated reprogramming transgenes in the iPS cell genome (solved by 2nd and 3rd generation reprogramming protocols that rely on non-integrative viral vectors or episomal plasmids, reviewed in [38], the propensity of iPS cells to silence disease-correcting transgenes (since disease correction can now in principle be achievable using recently developed techniques for targeted DNA editing, reviewed in [39], and the need to implement GMP-compliant protocols for the generation, culture and differentiation of iPS cells [reviewed in 40]. There remain, however, two major roadblocks to the clinical application of iPS-based cell replacement therapies. On the one hand, currently available protocols for the directed differentiation of specific cell types are, for the most part, rather inefficient and result in mixed cell populations, which typically are not mature enough to successfully graft and functionally integrate within the host's tissue or organ upon transplantation. Current iPS/ hES differentiation protocols are also difficult to scale up and to be adapted under GMP-compliant conditions. On the other hand, despite recent efforts to investigate the extent of genomic and epigenetic stability of iPS cells [reviewed in 41], there is no convincing evidence so far demonstrating that iPS-derived cells are not more susceptible to undergo malignant transformation than hES cells. Large-scale pre-clinical studies on the safety of different types of iPS-derived cells will be necessary to unambiguously address this issue, before clinical trials can be designed with the appropriate risk-benefit factor taken into account [42].

In any case, the scenario of developing patient-specific, autologous iPS-based cell replacement therapies, does not appear to be economically sustainable, or at least not for highly prevalent diseases. A more feasible alternative could come from the use of iPS-derived cells in an allogeneic setting, as banks of HLA-typed, GMP-grade and thoroughly tested iPS cell lines could be created so that they provided a good match for large segments of the population. The first of these banks is currently being set up in Japan with 75 iPS cell lines homozygous for the most common HLA haplotypes, which is expected to provide coverage for almost $80 \%$ of the Japanese population [43]. Irrespective of whether this particular bank will eventually turn out to be useful, or just prove to have been a premature move by the Japanese Ministry of Health, what it clearly shows is that the stakes of clinical translation of iPS cells are currently at an all-time high. Taking into consideration the pace at which we have witnessed iPS cell research advance over the last 6 years, it will not be too risky to forecast that real benefits for patients (in the form of cell therapy applications and/or novel drugs discovered thanks to iPS-based disease models) will be tangible in the very short term.

\section{ACKNOWLEDGEMENTS}

This work was supported by grants from the Ministry of Economy and Competitiveness (FIS PI10/02529, FIS EC07/90762, FIS PI12/00760, FIS PI13/00666), the Ministry of Science and Technology (BIO200913903-C02-02), and the Andalusian Government (P07-CVI-2781, PAIDI BIO-217, PI-0729-2010). Spanish Cell Therapy Network (TerCel) and CIBER-BBN are an initiative funded by the VI National R\&D\&I Plan 2008-2011 (RD06/0010/0023, RD12/0019/0001), Advanced Therapies and Transplant General Direction (Health Ministry, Spain) (TRA-137), 
Iniciativa Ingenio 2010, Consolider Program, CIBER Actions, and financed by the Instituto de Salud Carlos III (ISC-III) with assistance from the European Regional Development Fund. Work in MuñozChapuli's laboratory is supported by grants BFU2011-25304, BFU201235799, P11-CTS-7564, and PITN-GA-2011-289600; in Raya’s laboratory by grants SAF2012-33526, ACI2010-1117, and ISC-III (TerCel, RD12/0019/0019).

\section{REFERENCES}

[1] Sipp, D. (2011) Global challenges in stem cell research and the many roads ahead. Neuron, 70, 573-576. http://dx.doi.org/10.1016/j.neuron.2011.05.008

[2] Trounson, A. and Dewitt, N.D. (2012) Stem cell biology: Towards the reality of cell therapeutics. Nature Cell Biology, 14, 331. http://dx.doi.org/10.1038/ncb2469

[3] Mustafiz, T., Portelius, E., Gustavsson, M.K., et al. (2010) Characterization of the brain beta-amyloid isoform pattern at different ages of Tg2576 mice. Neurodegenerative Diseases, 8, 352-363. http://dx.doi.org/10.1159/000323871

[4] Kordower, J.H. (2008) Introduction to the special ASNTR issue. Cell Transplantation, 17, 361-362.

[5] Cicchetti, F., Drouin-Ouellet, J. and Gross, R.E. (2009) Environmental toxins and Parkinson's disease: What have we learned from pesticide-induced animal models? Trends of Pharmacology Science, 30, 475-483. http://dx.doi.org/10.1016/j.tips.2009.06.005

[6] Ekdahl, C.T., Kokaia, Z. and Lindvall, O. (2009) Brain inflammation and adult neurogenesis: The dual role of microglia. Neuroscience, 158, 1021-1029. http://dx.doi.org/10.1016/j.neuroscience.2008.06.052

[7] Arvidsson, L., Fagerlund, M., Jaff, N., et al. (2011) Distribution and characterization of progenitor cells within the human filum terminale. PloS One, 6, e27393. http://dx.doi.org/10.1371/journal.pone.0027393

[8] Ekdahl, C.T. (2012) Microglial activation-tuning and pruning adult neurogenesis. Frontier of Pharmacology, 3, 41. http://dx.doi.org/10.3389/fphar.2012.00041

[9] Winner, B., Kohl, Z. and Gage, F.H. (2011) Neurodegenerative disease and adult neurogenesis. European Journal of Neuroscience, 33, 1139-1151. http://dx.doi.org/10.1111/j.1460-9568.2011.07613.x

[10] Blanquer, M., Pérez-Espejo, M.A., Martínez-Lage, J.F., et al. (2010) A surgical technique of spinal cord cell transplantation in amyotrophic lateral sclerosis. Journal of Neuroscience Methods, 191, 255-257. http://dx.doi.org/10.1016/j.jneumeth.2010.06.014

[11] Blanquer, M., Moraleda, J.M., Iniesta, F., et al. (2012) Neurotrophic bone marrow celular nests prevent spinal motoneuron degeneration in amyotrophic lateral sclerosis patients: A pilot safety study. Stem Cells, 30, 1277-1285. http://dx.doi.org/10.1002/stem.1080

[12] Araque-Monrós, M.C., Gil-Santos, L., Monleón-Pradas, M. and Más-Estellés, J. (In press) New concept for a regenerative and resorbable prosthesis for tendon and ligament. Physicochemical and biological characterization of
PLA braided biomaterial. Journal of Biomedical Materials Research: Part A.

[13] Andrades, J.A., Motaung, S.C., Jiménez-Palomo, P., et al. (2012) Induction of superficial zone protein (SZP)/lubricin/PRG 4 in muscle-derived mesenchymal stem/progenitor cells by transforming growth factor-beta1 and bone morphogenetic protein-7. Arthritis Research and Therapy, 14, 31-42. http://dx.doi.org/10.1186/ar3793

[14] Granero-Moltó, F., Weis, J.A., Miga, M.I., et al. (2009) Regenerative effects of transplanted mesenchymal stem cells in fracture healing. Stem Cells, 27, 1887-1898. http://dx.doi.org/10.1002/stem.103

[15] Evans, C.H. (2013) Advances in regenerative orthopedics. Mayo Clinic Proceedings, 88, 1323-1339. http://dx.doi.org/10.1016/j.mayocp.2013.04.027

[16] Orozco, L., Soler, R., Morera, C., et al. (2011) Intervertebral disc repair by autologous mesenchymal bone marrow cells: A pilot study. Transplantation, 92, 822-828. http://dx.doi.org/10.1097/TP.0b013e3182298a15

[17] Gallego, L., Junquera, L., García, E., et al. (2010) Repair of rat mandibular bone defects by alveolar osteoblasts in a novel plasma-derived albumin scaffold. Tissue Engineering Part A, 16, 1179-1187. http://dx.doi.org/10.1089/ten.tea.2009.0517

[18] Le Blanc, K. and Ringden, O. (2007) Immunomodulation by mesenchymal stem cells and clinical experience. Journal of International Medicine, 262, 509-525. http://dx.doi.org/10.1111/j.1365-2796.2007.01844.x

[19] Chang, C.H., Kuo, T.F., Lin, F.H., et al. (2011) Tissue engineering-based cartilage repair with mesenchymal stem cells in a porcine model. Journal of Orthopaedic Research, 29, 1874-1880. http://dx.doi.org/10.1002/jor.21461

[20] Bernardo, M.E., Pagliara, D. and Locatelli, F. (2011) Mesenchymal stromal cell therapy: A revolution in Regenerative Medicine? Bone Marrow Transplantation, 47, 164-171. http://dx.doi.org/10.1038/bmt.2011.81

[21] Takahashi, K. and Yamanaka, S. (2006) Induction of pluripotent stem cells from mouse embryonic and adult fibroblast cultures by defined factors. Cell, 126, 663-676. http://dx.doi.org/10.1016/j.cell.2006.07.024

[22] Vogel, G. (2010) Stem cells. Reprogrammed cells come up short, for now. Science, 327, 1191-1197.

[23] Graf, T. and Enver, T. (2009) Forcing cells to change lineages. Nature, 462, 587-594. http://dx.doi.org/10.1038/nature08533

[24] Vierbuchen, T. and Wernig, M. (2011) Direct lineage conversions: Unnatural but useful? Nature Biotechnology, 29, 892-907. http://dx.doi.org/10.1038/nbt.1946

[25] Mizrak, S.C., Chikhovskaya, J.V., Sadri-Ardekani, H., et al. (2009) Embryonic stem cell-like cells derived from adult human testis. Human Reproduction, 25, 158-167. http://dx.doi.org/10.1093/humrep/dep354

[26] Aasen, T., Raya, A., Barrero, M.J., et al. (2008) Efficient and rapid generation of induced pluripotent stem cells from human keratinocytes. Nature Biotechnology, 26, 1276-1284. http://dx.doi.org/10.1038/nbt.1503

[27] Giorgetti, A., Montserrat, N., Aasen, T., et al. (2009) 
Generation of induced pluripotent stem cells from human cord blood using OCT4 and SOX2. Cell Stem Cell, 5, 353-357. http://dx.doi.org/10.1016/j.stem.2009.09.008

[28] Ebert, A.D., Yu, J., Rose, F.F., et al. (2009) Induced pluripotent stem cells from a spinal muscular atrophy patient. Nature, 457, 277-280.

http://dx.doi.org/10.1038/nature07677

[29] Lee, H., Park, J., Forget, B.G. and Gaines, P. (2009) Induced pluripotent stem cells in regenerative medicine: An argument for continued research on human embryonic stem cells. Regenerative Medicine, 4, 759-769. http://dx.doi.org/10.2217/rme.09.46

[30] Carvajal-Vergara, X., Sevilla, A., D’Souzza, S.L., et al. (2010) Patient-specific induced pluripotent stem-cell derived models of LEOPARD syndrome. Nature, 465, 808812. http://dx.doi.org/10.1038/nature09005

[31] Ku, S., Soragni, E., Campau, E., Thomas, E.A., Altun, G., Laurent, L.C., Loring, J.F., Napierala, M. and Gottesfeld, J.M. (2010) Friedreich's ataxia induced pluripotent stem cells model intergenerational GAA-TTC triplet repeat instability. Cell Stem Cell, 7, 631-637. http://dx.doi.org/10.1016/j.stem.2010.09.014

[32] Moretti, A., Bellin, M., Welling, A., et al. (2010) Patientspecific induced pluripotent stem-cell models for longQT syndrome. New England Journal of Medicine, 363, 1397-1409.

http://dx.doi.org/10.1056/NEJMoa0908679

[33] Rashid, S.T., Corbineau, S., Hannan, N., Marciniak, S.J., Miranda, E., Alexander, G., Huang-Doran, I., Griffin, J., Ahrlund-Richter, L., Skepper, J., Semple, R., Weber, A., Lomas, D.A. and Vallier, L. (2010) Modeling inherited metabolic disorders of the liver using human induced pluripotent stem cells. Journal of Clinical Investigation, 120, 3127-3136. http://dx.doi.org/10.1172/JCI43122

[34] Zhang, S., Chen, S., Li, W., Guo, X.P., Zhao, P., Xu, J.Y., Chen, Y., Pan, Q., Liu, X.R., Zychlinski, D., Lu, H., Tortorella, M.D., Schambach, A., Wang, Y., Pei, D.Q. and Esteban, M.A. (2011) Rescue of ATP7B function in hepatocyte-like cells from Wilson's disease induced pluripotent stem cells using gene therapy or the chaperone drug curcumin. Human Molecular Genetics, 20, 31763187. http://dx.doi.org/10.1093/hmg/ddr223

[35] Brennand, K.J., Simone, A., Jou, J., Gelboin-Burkhart, C.,
Tran, N., Sangar, S., Li, Y., Mu, Y.L., Chen, G., Yu, D., McCarthy, S., Sebat, J. and Gage, F.H. (2011) Modelling schizophrenia using human induced pluripotent stem cells. Nature, 473, 221-225.

http://dx.doi.org/10.1038/nature09915

[36] Sánchez-Danés, A., Richaud-Patin, Y., Carballo-Carbajal, I., Jiménez-Delgado, S., Caig, C., Mora, S., Di Guglielmo, C., Ezquerra, M., Patel, B., Giralt, A., Canals, J.M., Memo, M., Alberch, J., López-Barneo, J., Vila, M., Cuervo, A.M., Tolosa, E., Consiglio, A. and Raya, A. (2012) Diseasespecific phenotypes in dopamine neurons from human iPS-based models of genetic and sporadic Parkinson's disease. EMBO Molecular Medicine, 4, 380-395. http://dx.doi.org/10.1002/emmm.201200215

[37] Raya, A., Rodríguez-Pizá, I., Guenechea, G., Vassena, R., Navarro, S., Barrero, M.J., Consiglio, A., Castellà, M., Río, P., Sleep, E., González, F., Tiscornia, G., Garreta, E., Aasen, T., Veiga, A., Verma, I.M., Surrallés, J., Bueren, J. and Belmonte, J.C.I. (2009) Disease-corrected haematopoietic progenitors from Falconi anaemia induced pluripotent stem cells. Nature, 460, 53-59. http://dx.doi.org/10.1038/nature08129

[38] Ma, N., Liao, B., Zhang, H., et al. (2013) TALEN-mediated gene correction in integration-free-beta-thalassemia iPSCs. Journal of Biology and Chemistry, Epub ahead of print.

[39] Perez-Pinera, P., Ousterout, D.G. and Gersbach, C.A. (2012) Advances in targeted genome editing. Current Opinion in Chemical Biology, 16, 268-277. http://dx.doi.org/10.1016/j.cbpa.2012.06.007

[40] Okano, H., Nakamura, M., Yoshida, K., Okada, Y., Tsuji, O., Nori, S., Ikeda, E., Yamanaka, S. and Miura, K. (2013) Steps toward safe cell therapy using induced pluripotent stem cells. Circulation Research, 112, 523-533. http://dx.doi.org/10.1161/CIRCRESAHA.111.256149

[41] Ronen, D. and Benvenisty, N. (2012) Genomic stability in reprogramming. Current Opinion in Genetics \& Development, 22, 444-449.

http://dx.doi.org/10.1016/j.gde.2012.09.003

[42] Cyranoski, D. (2013) Stem cells cruise to clinic. Nature, 494, 413. http://dx.doi.org/10.1038/494413a

[43] Cyranoski, D. (2012) Stem-cell pioneer banks on future therapies. Nature, 488, 139. http://dx.doi.org/10.1038/488139a 